CASTROVIEJO, S., M. LAÍNZ, G. LÓPEZ GONZÁLEZ, P. MONTSERRAT, F. MUÑOZ GARMENDIA, J. PAIVA y L. VILLAR -1986/ 1993- [eds.] Flora Iberica. C.S.I.C. Madrid.

CLEMENT, B. \& J. TOUFFET -1978- Les pelouses xerophiles autour de quelques affleurements schisteux en Bretagne interieure. Colloques Phytosociologiques, 6: 177-189.

DÍAZ GONZÁLEZ, T. E. y J.A. FERNÁNDEZ PRIETO -1994- La vegetación de Asturias. Itinera Geobot., 8: 243-528.

GÉHU, J.M., J. GÉHU \& B. CARON -1978- Les groupements á Sedum anglicum des falaises Nord-Armoricaines. Colloques Phytosociologiques, 6: 255-261.

MORALES, R. -1986- Taxonomía de los géneros Thymus (excluída la sección Serpyllium) y Thymbra en la península Ibérica. Ruizia, 3: (1324).

RIVAS-MARTÍNEZ, S. -1987-Memoria del mapa de las series de vegetación de España
(1:400.000). ICONA, Serie Técnica. Madrid.

RIVAS-MARTÍNEZ, S. y P. CANTÓ -1987- Datos sobre la vegetación de las sierras de Guadarrama y Malagón. Lazaroa, 7: 235-257.

SMITH, A. -1980- The moss flora of Britain and Ireland. Cambridge University Press. Cambridge.

TUTIN, T.G., V.H. HEYWOOD, N.A. BURGES, D.H. VALENTINE, S.M. WALTERS \& D.A. WEBB -(1964-1980)- [eds.] Flora europea. Vol. 1-5. Cambridge.

Aceptado para su publicación en Julio de 1996

Dirección de los autores. Dpto. Biología Vegetal. Laboratorio de Botánica. Facultad de Farmacia. Campus Universitario Sur. 15706, Santiago de Compostela

\title{
56. STIPION PARVIFLORAE ALL. NOVA, PASTIZALES VIVACES SUBNITRÓFILOS Y CALCÍCOLAS MEDITERRÁNEO-IBEROLEVANTINOS
}

\author{
Antonio DE LA TORRE, Francisco ALCARAZ y María VICEDO
}

Stipion parviflorae all. nova, perennial subnitrophilous and calcicolous mediterraneaniberolevantines grassland

Palabras clave: Fitosociología, ibero-levantino, Stipion parviflorae all. nova, Lygeo-Stipetea, pastizales.

Key words: Phytosociology, ibero-levantine, Stipion parviflorae all. nova, Lygeo-Stipetea, grassland.

Dentro de la clase Lygeo-Stipetea RivasMartínez 1978 se han venido reconociendo, desde que Rivas-Martínez (1978) propusiera el orden subnitrófilo Hyparrhenietalia hirtae, una serie de comunidades dominadas por hemicriptófitos herbáceos en las que abundan las gramíneas del género Stipa.

Estos pastizales viven sobre suelos poco profundos o muy alterados por acción antrópica o erosión, siempre en áreas térmicas, en los 
que se advierte cierta nitrificación. Juegan el papel de auténticas comunidades pioneras, colonizando sustratos que están desprovistos de vegetación perenne y favoreciendo la posterior instalación de otras que requieren un sustrato más estabilizado.

Dada la composición florística, se trata de asociaciones que podrían pertenecer al orden Thero-Brachypodietalia (Br.-Bl. 1931) Molinier 1934, pero si admitimos un orden para las comunidades pioneras subnitrófilas o de suelos esqueléticos dentro de la clase LygeoStipetea, es obvio que parece más acertada su ubicación en Hyparrhenietalia hirtae. E1 carácter subnitrófilo de estas comunidades se ve reflejado también en el hecho de que cuentan con muchas especies nitrófilas de las clases Artemisietea vulgaris Lohmeyer, Preising et R.Tx. 1950 ampl. Rivas-Martínez in RivasMartínez et al. 1991 y Pegano-Salsoletea Br.Bl. et O. Bolòs 1958 .

Con el fin de englobar este tipo de comunidades se propone la nueva alianza Stipion parviflorae.

Stipion parviflorae De la Torre, Alcaraz et Vicedo all. nova

Holotypus: Plantagini albicantisStipetum parviflorae ass. nova

Asociaciones calcícolas dominadas por especies vivaces subnitrófilas en las que abundan las gramíneas del género Stipa. Se desarrollan en la superprovincia Mediterráneoiberolevantina, en los termotipos termo y mesomediterráneo bajo ombroclima semiárido y seco.

Consideramos características de esta alianza las siguientes especies: Stipa parviflora, S. celakovskyi, S. barbata, S. iberica subsp. pauneroana, S. lagascae (ass.), Plantago albicans (óp.).

Son frecuentes otras especies de otras unidades de la clase, tales como Eryngium campestre, Koeleria vallesiana, Sedum sediforme, Brachypodium retusum, Dactylis hispanica, Phlomis lychnitis, Convolvulus lineatus, Asphodelus ramosus, Lygeum spartum, etc.

Dentro del orden Hyparrhenietalia hirtae Rivas-Martínez 1978 se incluyen las alianzas Dauco-Hyparrhenion hirtae Br.-Bl., Pinto da Silva et Rozeira 1956, Micromerio graecaeHyparrhenion sinaicae O. Bolòs 1962 corr. y Stipion parviflorae all nova. La primera de ellas es de distribución ibero-atlántica, frente a las dos restantes de distribución iberolevantina. Las alianzas MicromerioHyparrhenion y Stipion parviflorae difieren fundamentalmente en su ecología. Mientras que la primera de ellas es propia de laderas pedregosas con buen drenaje, la segunda se desarrolla sobre suelos compactados, nunca en pendientes acusadas.

Dentro de esta nueva alianza se incluyen, a nuestro parecer, las siguientes asociaciones, de las cuales la primera la proponemos como nueva.

Plantagini albicantis-Stipetum parviflorae ass. nova

Lygeo-Stipetum lagascae $\mathrm{Br} . \mathrm{Bl}$. et $\mathrm{O}$. Bolòs 1957

Brachypodio-Stipetum pennatae O. Bolòs 1954

\section{* Plantagini albicantis-Stipetum parviflorae ass. nova}

(Tabla 1. Holotypus: inv. 5)

Asociación de los termotipos termomediterráneo y horizonte inferior del mesomediterráneo, bajo ombroclima semiárido. De acuerdo con las indicaciones biogeográficas propuestas por Peinado et Rivas-Martínez (1987), Alcaraz et al.(1991) y De la Torre et al. (en prensa), esta asociación presenta su óptimo en la provincia MurcianoAlmeriense, pero llega a los enclaves más áridos y térmicos de los subsectores Ayorano- 


\begin{tabular}{lcccccccccc}
\hline Altitud (Dm) & 36 & 40 & 52 & 20 & 30 & 52 & 15 & 20 & 64 & 20 \\
Area (m2) & 50 & 100 & 100 & 10 & 60 & 20 & 50 & 20 & 15 & 10 \\
Núm. Especies & 19 & 13 & 12 & 8 & 13 & 5 & 10 & 9 & 6 & 7 \\
Núm. Orden & 1 & 2 & 3 & 4 & 5 & 6 & 7 & 8 & 9 & 10 \\
\hline
\end{tabular}

Combinación habitual

$\begin{array}{lcccccccccc}\text { Stipa parviflora } & 3.2 & 3.4 & 3.3 & 3.3 & 4.4 & 3.3 & 3.3 & 4.4 & 4.4 & 3.3 \\ \text { Plantago albicans } & 2.1 & + & . & + & 1.1 & + & + & + & 1.1 & + \\ \text { Dactylis hispanica } & . & + & +.2 & 1.2 & 1.2 & 4.4 & 1.2 & . & 1.2 & +.2 \\ \text { Asphodelus fistulosus } & 1.1 & + & . & 2.2 & 2.3 & . & 2.2 & 1.1 & . & 1.1 \\ \text { Convolvulus althaeoides } & + & . & . & . & 1.1 & . & 1.1 & 1.1 & . & .\end{array}$

Características de la alianza, orden y clase

Brachypodium retusum

Asphodelus ramosus

Sedum sediforme

Phagnalon saxatile

Bituminaria bituminosa

Hyparrhenia sinaica

Lygeum spartum

\section{Compañeras}

Paronychia capitata

Reichardia tingitana

Teucrium gracillimum

Andryala ragusina

Asteriscus maritimus

Eryngium campestre

Fumana thymifolia

Helianthemum violaceum

Helichrysum stoechas

Piptatherum miliaceum

Salsola genistoides

Salvia verbenaca

$\begin{array}{cccc} & +.2 & . & +.2 \\ . & 1.1 & \cdot & + \\ 1.1 & 1.1 & . & + \\ . & \cdot & + & . \\ +.2 & . & . & .\end{array}$

$\begin{array}{ccc} & \cdot & +2 \\ \cdot & \cdot & + \\ + & \cdot & + \\ \cdot & \cdot & \cdot \\ \cdot & \cdot & \cdot \\ \cdot & \cdot & \cdot \\ . & . & .\end{array}$

3.3

1.1

$\begin{array}{ccc}. & 3.3 & . \\ . & \cdot & 1.1 \\ 1.1 & \cdot & \cdot \\ \cdot & \cdot & \cdot \\ . & \cdot & \cdot \\ +.2 & \cdot & .\end{array}$

$\begin{array}{cccc}1.1 & . & 1.1 & + \\ 1.1 & 1.1 & \cdot & \cdot \\ 1.1 & 1.1 & \cdot & \cdot \\ + & \cdot & + & \cdot \\ . & \cdot & \cdot & \cdot \\ + & \cdot & \cdot & \cdot \\ 1.2 & . & \cdot & \cdot \\ + & 1.1 & \cdot & \cdot \\ 1.1 & \cdot & + & \cdot \\ + & . & \cdot & \cdot \\ + & + & . & .\end{array}$

\section{EN UN SOLO INVENTARIO:}

Compañeras: Ajuga iva + en el inv. 5. Anthyllis cytisoides + en el inv. 2. Artemisia glutinosa + en el inv. 3. Asparagus horridus + en el inv. 5. Centaurea aspera + en el inv. 1. Cynodon dactylon 2.2 en el inv. 3. Euphorbia segetalis + en el inv. 5. Euphorbia serrata + en el inv. 1. Hammada articulata 2.2 en el inv. 2. Imperata cylindrica + en el inv. 3. Lobularia maritima 1.1 en el inv. 3. Medicago truncatula + en el inv. 6. Moricandia arvensis + en el inv. 9. Pallenis spinosa 1.1 en el inv. 8. Phagnalon rupestre 1.2 en el inv. 10. Sonchus tenerrimus + en el inv. 5. Sorghum halepense + en el inv. 3. Thymelaea hirsuta 1.1 en el inv. 1. Thymus vulgaris + en el inv. 1 .

Procedencia de los inventarios: 1.- Casas de Abajo, Agost. 2.- Casas de Miras, Agost. 3.- Arenales de las Virtudes, Villena. 4.- Punta Prima, Orihuela. 5.- La Zenia, Orihuela (Holotypus). 6.- Ctra. Sax-Castalla. 7.- La Zenia, Orihuela. 8.- El Moralet, San Vicente. 9.- Pusa, Petrer. 10.- La Matanza, Santomera.

Tabla 1. Ass. Plantagini albicantis-Stipetum parviflorae De la Torre, Alcaraz et Vicedo nova. 
Villenense, Alcoyano-Diánico, ManchegoMurciano y Manchego-Espunense. Se caracteriza por la presencia de especies xerotermófilas que rehuyen la continentalidad.

Se desarrolla sobre suelos compactados en terrenos que han sido removidos y posteriormente abandonados, así como en los taludes entre cultivos. Los suelos sobre la que la hemos podido observar son calcisoles pétricos o háplicos y regosoles calcáricos o gípsicos.

Cuando un terreno de labor. es periódicamente arado no pueden asentarse plantas vivaces y las comunidades que aparecen pertenecen al suborden Sisymbrienalia officinalis J. Tüxen in Lohmeyer et al. 1962 em. Rivas-Martínez et al. 1991. Si el mismo terreno es abandonado se observa una sustitución progresiva de las comunidades de Sisymbrienalia officinalis por otras de Bromenalia rubenti-tectorum Rivas-Martínez et Izco 1977. Con el tiempo, a partir del segundo año sin alteraciones, el campo abandonado se puebla de pastizales vivaces correspondientes a la alianza Stipion parviflorae que se propone. Por excesivo pisoteo se remplaza por la asociación Salvio-Plantaginetum albicantis $\mathrm{O}$. Bolòs et R. Molinier 1984, que supone el preludio a los matorrales nitrófilos del orden Salsolo-Peganetalia Br.-Bl. et O.Bolòs 1954. En las áreas semiáridas del territorio estudiado, la sucesión es:

Eruco longirostris-Diplotaxietum erucoidis Rigual 1972 corr. Alcaraz 1984

Bromo fasciculati-Aegilopetum geniculatae Sánchez-Gómez et al. 1992

Plantagini-Stipetum parviflorae De la Torre et al. ass. nova

Salvio-Plantaginetum albicantis 0 . Bolòs et $\mathrm{R}$. Molinier 1984

Thymelaeo-Artemisietum barrelieri Alcaraz et al. 1991
Se diferencia de la asociación litoral catalana (Brachypodio-Stipetum pennatae) por la falta de elementos mesofíticos como Stipa capillata, Stipa pauneroana, Festuca duriuscula, etc. De la asociación aragonesa queda diferenciada por la presencia de especies termófilas como Convolvulus althaeoides, Asphodelus fistulosus, Asteriscus maritimus, Ajuga iva o Asparagus horridus. Si bien con esta última se encuentra más relacionada, dada la presencia en las dos de Stipa parviflora frente a $S$. capillata y $S$. pauneroana propias de los pastizales catalanes del BrachypodioStipetum pennatae.

* Lygeo-Stipetum lagascae Br.-Bl. et $\mathrm{O}$. Bolòs in Anales Estac. Exper. Aula Dei, vol. 5 (1957), invs. 1 a 7, tab. 27 (Lectotypus: loc. cit., inv. 4, tab. 27).

Asociación aragonesa caracterizada por Stipa lagascae, propia de los territorios continentales con tendencia semiárida.

Braun-Blanquet et Bolòs (1957) describen la asociación Lygeo-Stipetum lagascae que incluyen en la alianza Eremopyro cristatiLygeion sparti Br.-Bl. et O. Bolòs 1958 em. Rivas-Martínez 1978. La mayor parte de los inventarios existentes corresponden a albardinales que, por pertenecer a la asociación Eremopyro-Lygeetum Br.-B1. et O. Bolòs 1957 , se incluirían en esta alianza. Sin embargo los inventarios 1-7 presentan una combinación florística y fisonomía similar a la de otras asociaciones que se proponen incluir en la alianza Stipion parviflorae.

Transcripción del inventario designado como tipo de la asociación, eliminando los terófitos:

Stipa lagascae 5.5, Plantago albicans + , Stipa parviflora +.2, Centaurea melitensis 1.1, Poa bulbosa +, Dactylis hispanica 1.2, Eryngium campestre +, Stipa barbata + , Artemisia valentina + , Peganum harmala,+ Taraxacum sp. + , Koeleria vallesiana + . 


\begin{tabular}{|c|c|c|c|c|c|c|c|c|c|}
\hline $\begin{array}{l}\mathrm{N}^{0} \text { de inventarios } \\
\mathrm{N}^{o} \text { de orden }\end{array}$ & $\begin{array}{l}5 \\
1\end{array}$ & $\begin{array}{c}10 \\
2\end{array}$ & $\begin{array}{l}4 \\
3\end{array}$ & $\begin{array}{c}10 \\
4\end{array}$ & $\begin{array}{l}\mathrm{N}^{\circ} \text { de inventarios } \\
\mathrm{N}^{0} \text { de orden }\end{array}$ & $\begin{array}{l}5 \\
1\end{array}$ & $\begin{array}{c}10 \\
2\end{array}$ & $\begin{array}{l}4 \\
3\end{array}$ & $\begin{array}{r}10 \\
4\end{array}$ \\
\hline Atractylis humilis & 1 & . & . & . & Convolvulus cantabrica & . & . & IV & \\
\hline Centaurea melitensis & 5 & . & . & . & Festuca ovina ssp. & . & . & III & \\
\hline Elymus hispidus & 3 & . & . & . & Sanguisorba muricata & . & . & . & I \\
\hline Erucastrum nasturtiifolium & 1 & . & . & . & Trinia glauca & . & . & IV & \\
\hline Leontodon hispanicus & 2 & . & . & & Anthyllis font-queri & . & . & . & 11 \\
\hline Mantisalca salmantica & 1 & . & . & . & Aphyllanthes monspeliensis & . & . & . & II \\
\hline Ononis pusilla & 1 & . & . & & Avenula bromoides & . & . & . & IV \\
\hline Orobanche cernua & 1 & . & . & . & Bromus erectus & . & . & . & I \\
\hline Poa bulbosa & 3 & . & . & . & Carduncellus monspelliensium & & . & & \\
\hline Stipa barbata & 1 & . & . & . & Carex caryophyllea & . & . & III & \\
\hline Stipa lagascae & 5 & . & . & . & Carex humilis & . & . & . & \\
\hline Teucrium gnaphalodes & 1 & . & . & . & Centaurea pinae & . & . & . & IV \\
\hline Thymus zygis & 1 & & . & . & Coronilla minima & . & . & . & II \\
\hline Ajuga iva & . & I & . & . & Echinops ritro & . & . & . & III \\
\hline Andryala ragusina & . & I & . & . & Erysimum grandiflorum & . & . & . & III \\
\hline Anthyllis cytisoides & . & I & . & . & Euphorbia nicaensis & . & . & . & II \\
\hline Asphodelus fistulosus & . & IV & . & . & Festuca hervieri & . & . & . & \\
\hline Asteriscus maritimus & . & I & . & . & Fumana procumbens & . & . & . & II \\
\hline Bituminaria bituminoides & . & I & . & . & Galium gr. Lucidum & . & . & . & I \\
\hline Convolvulus altaheoides & . & II & . & . & Hieracium pilosella & . & . & . & I \\
\hline Hammada articulata & . & I & 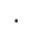 & 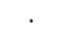 & Jurinea humilis & . & & . & I \\
\hline Hyparrhenia sinaica & . & I & 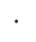 & . & Knutia subscaposa & . & . & . & I \\
\hline Lobularia maritima & . & I & 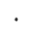 & 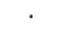 & Leuzea conifera & . & . & . & III \\
\hline Moricandia arvensis & . & I & 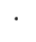 & · & Linaria aragonensis & . & . & . & II \\
\hline Pallenis spinosa & $\cdot$ & I & . & & Muscari comosum & . & . & . & I \\
\hline Paronychia capitata & $\cdot$ & II & . & - & Potentilla tabernamontani & . & . & . & II \\
\hline Phagnalon rupestre & $\cdot$ & I & . & & Santolina chamaecyparissus & . & . & III & \\
\hline Phagnalon saxatile & $\cdot$ & $\begin{array}{l}\text { II } \\
\text { I }\end{array}$ & . & . & Scabiosa turolensis & . & . & . & I \\
\hline $\begin{array}{l}\text { Salsola genistoides } \\
\text { Teucrium gracillimum }\end{array}$ & $\cdot$ & $\begin{array}{l}1 \\
I\end{array}$ & r. & & Stipa offneri & . & . & . & I \\
\hline Thymelaea hirsuta & & I & & & Teucrium chamaedrys & . & & . & II \\
\hline Sideritis hirsuta & & . & V & II & Teucrium expasum & 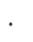 & . & & III \\
\hline Stipa capillata & & . & II & II & Teucrium polium & . & . & IV & \\
\hline Stipa pauneroana & & . & V & IV & Thalictrum tuberosum & & & 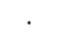 & II \\
\hline
\end{tabular}

1. Lygeo-Stipetum lagascae (Br.-B1. et O. Bolòs 1957, tabla 27, inv. 1-5 (se han eliminado los inventarios 6 y 7 por ser muy pobres en especies y del 8-30 por tratarse de albardinales)).

2. Plantagini albicantis-Stipetum parviflorae ass. nova (tabla 1).

3. Brachypodio-Stipetum pennatae (O. Bolòs 1983, pág. 63).

4. Thero-Stipetum sensu Aguilella non Vigo 1968. (Aguilella 1985, tabla 55)

Tabla 2. Tabla sintética de la Alianza Stipion parviflorae.

* Brachypodio-Stipetum pennatae 0 . Bolòs in Collect. Bot. (Barcelona), 4: 258-260 (1954), tab. 4 (Lectotypus: loc. cit., inv. 2, tab. 4).

Asociación catalano-provenzal caracterizada por Stipa pennata s.l., propia de ambientes frescos de la montaña. litoral catalana.
Transcripción del inventario designado como tipo de la asociación, eliminando los terófitos:

Allium sphaerocephalon + , Arenaria conimbricensis 2.1, Centaurea paniculata + , Convolvulus cantabrica + , Dipcadi serotinum + , Dorycnium pentaphyllum + , Eryngium campestre + , Fumana ericoides + , Helianthemum apenninum 
+ , Helianthemum italicum 1.1, Helichrysum stoechas + , Koeleria vallesiana 1.1 , Leontodon saxatilis 3.1, Potentilla verna + , Sanguisorba muricata + , Sedum sediforme +, Sideritis hirsuta 1.2, Stipa pennata 4.2, Teucrium capitatum +, Thymus vulgaris +, Trinia glauca + .

Relacionada con estas asociaciones se encuentra el pastizal Thero-Stipetum J. Vigo 1968, descrita como una comunidad de la que forman parte algunas especies de tendencia esteparia como Stipa pauneroana o Stipa capillata, de la que el autor señala su afinidad con el Brachypodio-Stipetum pennatae descrito por Bolòs (Vigo, 1968). Los inventarios aportados por el autor corresponden a pastizales de Brachypodium retusum por lo que se cree conveniente no incluir esta asociación en la alianza propuesta.

Los inventarios de Aguilella (1985) adscritos a la asociación Thero-Stipetum J. Vigo 1968, se pueden sinonimizar a la asociación anteriormente citada BrachypodioStipetum pennatae, dada la presencia en estos inventarios de Stipa capillata y S. pauneroana.

En la tabla 2 se presentan de forma destacada las especies diferenciales de las asociaciones mencionadas.

Las autorías de las especies que se recogen en las tablas se corresponden con las que figuran en Flora Iberica (Castroviejo et al., 1990-1993), Med-Checklist (Greuter et al, 1984-1989), Flora de Andalucía Occidental (Valdés et al, 1987) y Flora Europaea (Tutin et al., 1964-80), concediendo prioridad a la más reciente.

De acuerdo con lo anteriomente comentado, el esquema sintaxonómico quedaría de la siguiente forma:

\section{LYGEO SPARTI-STIPETEA RIVAS-MARTÍNEZ 1978}

+ Hyparrhenietalia hirtae Rivas-Martínez 1978

* Stipion parviflorae De la Torre, Alcaraz et Vicedo all. nova.

Lygeo-Stipetum lagascae Br.-Bl. et O. Bolòs 1957
Brachypodio-Stipetum pennatae O. Bolòs 1954

Plantagini albicantis-Stipetum parviflorae

De la Torre, Alcaraz et Vicedo ass. nova

\section{BIBLIOGRAFÍA}

ALCARAZ, F., P. SÁNCHEZ-GÓMEZ y A. DE LA TORRE -1991-. Biogeografía de la provincia Murciano-Almeriense hasta el nivel de subsector. Rivasgodaya, 6: 77-100.

AGUILELLA, A. -1985-. Flora y vegetación de la sierra del Toro y las Navas de Torrijas. Tesis doctoral inédita.

BOLÒs, O. -1954-. De vegetatione notulae, I. Collect. Bot. (Barcelona), 4: 258-260.

BOLÒS, O. -1983-. La vegetació del Montseny. Diputació de Barcelona, Servei de Parcs Naturals.

BRAUN-BLANQUET, J. y O. BOLÒS -1957-. Las comunidades vegetales de la depresión del Ebro y su dinamismo. Anales Estac. Exper. Aula Dei, 5(1-4): 1-266.

DE LA TORRE, A., F. ALCARAZ y M.B. CRESPO (en prensa). Aproximación a la biogeografía del sector Setabense (provincia CatalanoValenciano-Provenzal). Lazaroa, (en prensa).

PEINADO, M. y S. RIVAS-MARTÍNEZ (Eds.) 1987-. La vegeteción de España. Servicio de Publicaciones de la Universidad de Alcalá de Henares.

RIVAS-MARTÍNEZ, S. - 1978-. Sur la syntaxonomie des pelouses therophytiques de l'Europe occidentale. Colloques Phytosociol., 6: 55-71.

VIGO, J. -1968-. La vegetació del massís de Penyagolosa. Institut d'estudis catalans. Arxius de la secció de Ciències. XXXVII.

Aceptado para su publicación en Mayo de 1996

Dirección de los autores. A. de la Torre y M. Vicedo: Depto. de Ciencias Ambientales y Recursos Naturales (Botánica). Universidad de Alicante. Apartado 99, 03080. Alicante. F. Alcaraz: Depto. Biología Vegetal, Universidad de Murcia, 30100 Murcia. 